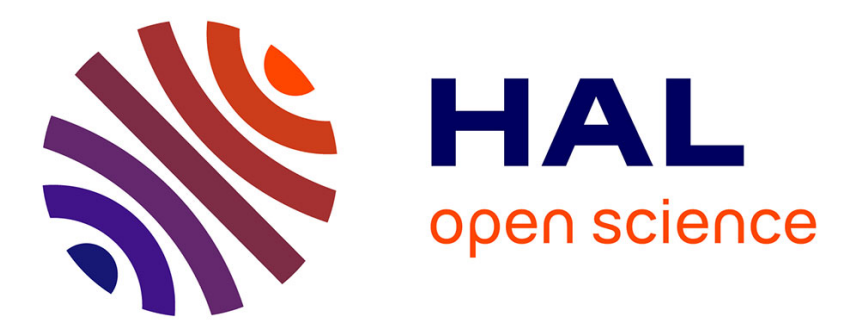

\title{
Réacteur de synthèse et de trempe dans un plasma hors d'équilibre: application à la synthèse de $\mathrm{C} 2 \mathrm{H} 2$ et $\mathrm{HCN}$
}

D. Rapakoulias, J. Amouroux

\section{To cite this version:}

D. Rapakoulias, J. Amouroux. Réacteur de synthèse et de trempe dans un plasma hors d'équilibre: application à la synthèse de C2H2 et HCN. Revue de Physique Appliquée, 1979, 14 (12), pp.961-968. 10.1051/rphysap:019790014012096100 . jpa-00244686

\section{HAL Id: jpa-00244686 https://hal.science/jpa-00244686}

Submitted on 1 Jan 1979

HAL is a multi-disciplinary open access archive for the deposit and dissemination of scientific research documents, whether they are published or not. The documents may come from teaching and research institutions in France or abroad, or from public or private research centers.
L'archive ouverte pluridisciplinaire HAL, est destinée au dépôt et à la diffusion de documents scientifiques de niveau recherche, publiés ou non, émanant des établissements d'enseignement et de recherche français ou étrangers, des laboratoires publics ou privés. 


\title{
Réacteur de synthèse et de trempe dans un plasma hors d'équilibre : application à la synthèse de $\mathrm{C}_{2} \mathrm{H}_{2}$ et $\mathrm{HCN}$
}

\author{
D. Rapakoulias et J. Amouroux \\ Laboratoire de Génie Chimique, E.N.S.C.P., 11, rue Pierre-et-Marie-Curie, 75231 Paris Cedex 05, France
}

(Reçu le 2 juillet, accepté le 24 septembre 1979)

\begin{abstract}
Résumé. - Dans cette étude nous proposons une nouvelle méthode d'analyse complète d'un réacteur chimique à plasma hors d'équilibre. Elle est basée sur l'association de l'observation expérimentale de la réactivité chimique, de l'analyse spectroscopique du plasma, les résultats des travaux en physique moléculaire et de la théorie des réactions en milieu hors d'équilibre.
\end{abstract}

Abstract. - Our aim is to propose a new method for the complete analysis of a non-equilibrium plasma chemical reactor. This method is based on the association of experimental study of reactors's chemical reactivity, spectroscopic analysis of plasma emission, data from molecular physics, and non-equilibrium reaction theory.

I. Introduction. - Dans le cas des oxydes d'azote, l'analyse du réacteur de synthèse hors d'équilibre en phase plasma a permis de préciser certaines notions sur la réaction chimique d'un plasma d'azote et son aptitude à permettre la fixation de l'azote.

Cette étude a pour objectif de proposer une nouvelle méthode d'analyse complète d'un réacteur chimique à plasma, basée sur l'association d'une observation expérimentale systématique et des dernières découvertes de la physique moléculaire et de la réactivité en milieu hors d'équilibre. Pour la mener à bien il s'agissait de choisir un modèle expérimental riche en information et nous avons entrepris l'étude du système méthane-azote qui autorise simultanément la synthèse de $\mathrm{C}_{2} \mathrm{H}_{2}$ et $\mathrm{HCN}$ c'est-à-dire deux réactions simultanées dont la vitesse dépend des caractéristiques du plasma d'azote.

Ainsi la première partie de l'étude consiste à trouver les corrélations entre les paramètres macroscopiques de marche du réacteur (pression, débits, énergie fournie) et les réactions élémentaires du plasma hors d'équilibre.

La deuxième partie consiste à dégager parmi les processus élémentaires ceux qui sont déterminants pour la formation des produits recherchés et en particulier à établir une corrélation entre les caractéristiques du plasma et la cinétique des processus hors d'équilibre.

Enfin nous conclurons par une présentation complète des processus de fixation de l'azote dans les deux cas étudiés et soulignant la différence de rôle des deux réacteurs de synthèse et des réacteurs de trempe suivant le type de liaison chimique établie : liaison $\mathrm{N}-\mathrm{C}$ et $\mathrm{N}-\mathrm{O}$.

II. Etude expérimentale. - Le dispositif expérimental a été décrit dans l'article précédent. Le plasma d'azote est créé par une décharge H.F. (38 MHz)à pression réduite ( 5 à 40 torr). Le méthane est introduit froid au niveau de la paroi de trempe, à contre-courant du plasma d'azote. La zone de mélange de deux gaz est à la fois la zone réactionnelle et la zone de trempe. Les gaz sortant du réacteur sont analysés par chromatographie [12], et ce résultat, confronté à l'analyse spectroscopique du plasma d'azote décrite dans l'article précédent, permet d'appréhender les voies de fixation de l'azote dans le réacteur.

En effet le système $\mathrm{N}_{2}-\mathrm{CH}_{4}$ comporte comme particularité réactionnelle le fait que deux réactions sont simultanément possibles :

* Réaction de transfert d'excitation entre l'azote excité et le méthane froid avec formation d'acétylène.

* Réaction de fixation de l'azote, avec formation d'acide cyanhydrique :

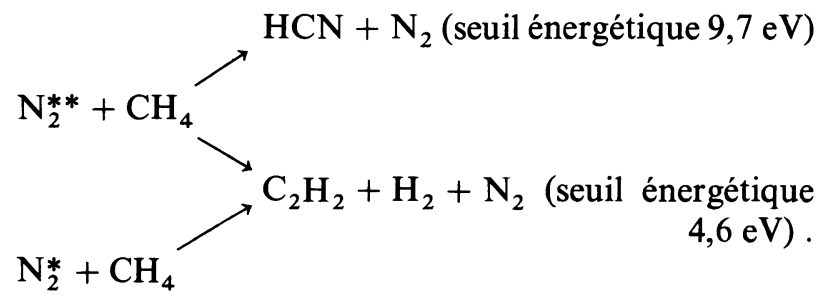


Il s'agit, bien entendu de réactions globales procédant par plusieurs étapes. (Nous avons désigné par $\mathrm{N}_{2}^{* *}$ les états excités de l'azote qui contiennent au moins $9,7 \mathrm{eV}$. Ces états peuvent être les $\mathrm{C}^{3} \Pi_{\mathrm{u}}$, $\mathrm{B}^{3} \Pi_{\mathrm{g}}$, en prédissociation, l'ion $\mathrm{N}_{2}^{+}$ou l'azote atomique. Le symbole $\mathrm{N}_{2}^{*}$ désigne un état excité contenant entre 4,6 et $9,7 \mathrm{eV}$. Un tel état peut être le métastable $\mathrm{A}^{3} \Sigma_{\mathrm{u}}^{+}$, ou le fondamental fortement excité vibrationnellement.)

II.1 INFLUENCE DE L'éNERGIE INDUITE (Fig. 1). Les résultats obtenus sont illustrés sur la figure 1 . Nous pouvons constater que l'augmentation d'énergie induite

a) favorise la décomposition du $\mathrm{CH}_{4}$,

b) à faible taux de conversion du méthane produit une augmentation simultanée de $\mathrm{HCN}$ et $\mathrm{C}_{2} \mathrm{H}_{2}$,

c) à fort taux de conversion du $\mathrm{CH}_{4}$ produit une augmentation de $\mathrm{HCN}$ et une diminution de $\mathrm{C}_{2} \mathrm{H}_{2}$, fixé.

d) permet une augmentation de la teneur en $\mathrm{N}_{2}$

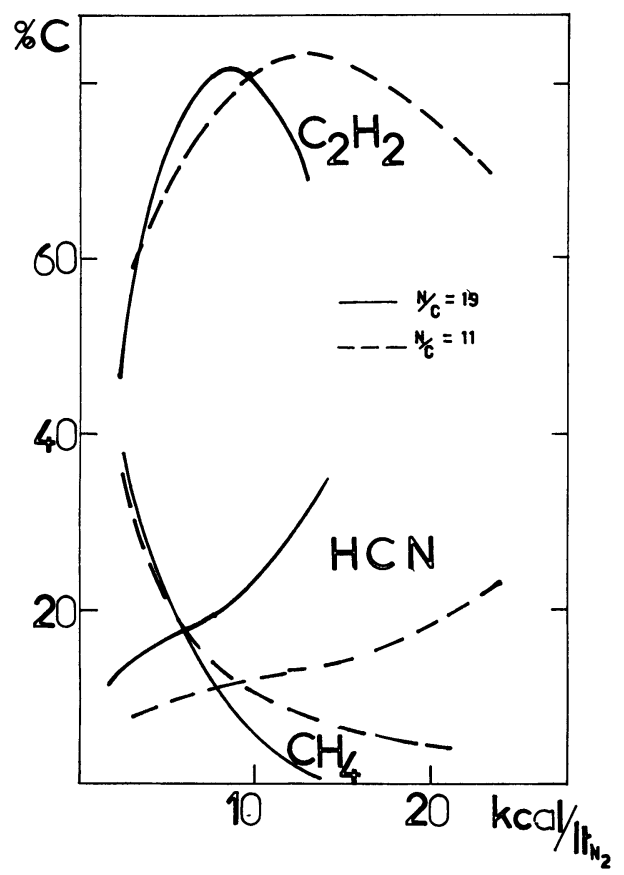

Fig. 1. - Taux de transformation du méthane initial en $\mathrm{C}_{2} \mathrm{H}_{2}$ et $\mathrm{HCN}$ en fonction de l'énergie induite, exprimée en kcal/l de $\mathrm{N}_{2}$. $P=10-13$ torrs. Débits $\mathrm{CH}_{4}$ et $\mathrm{N}_{2}$ constants.

[Conversion rate of methane to $\mathrm{C}_{2} \mathrm{H}_{2}$ and $\mathrm{HCN}$ as a function of induced energy.]

II. 2 INFLUENCE DU DÉBIT DE $\mathrm{N}_{2}$ (Fig. 2). - Une augmentation du débit de $\mathrm{N}_{2}$ produit les conséquences suivantes :

a) $\mathrm{Le} \mathrm{CH}_{4}$ disparaît progressivement des produits de la réaction. Quand tout le $\mathrm{CH}_{4}$ est transformé, le taux de $\mathrm{HCN}$ continue d'augmenter, au détriment de $\mathrm{C}_{2} \mathrm{H}_{2}$. Ces modifications sont toutefois faibles et dues essentiellement à l'augmentation de la vitesse de la réaction $\mathrm{N}_{2} \rightarrow \mathrm{CH}_{4}$ avec la concentration en $\mathrm{N}_{2}$.

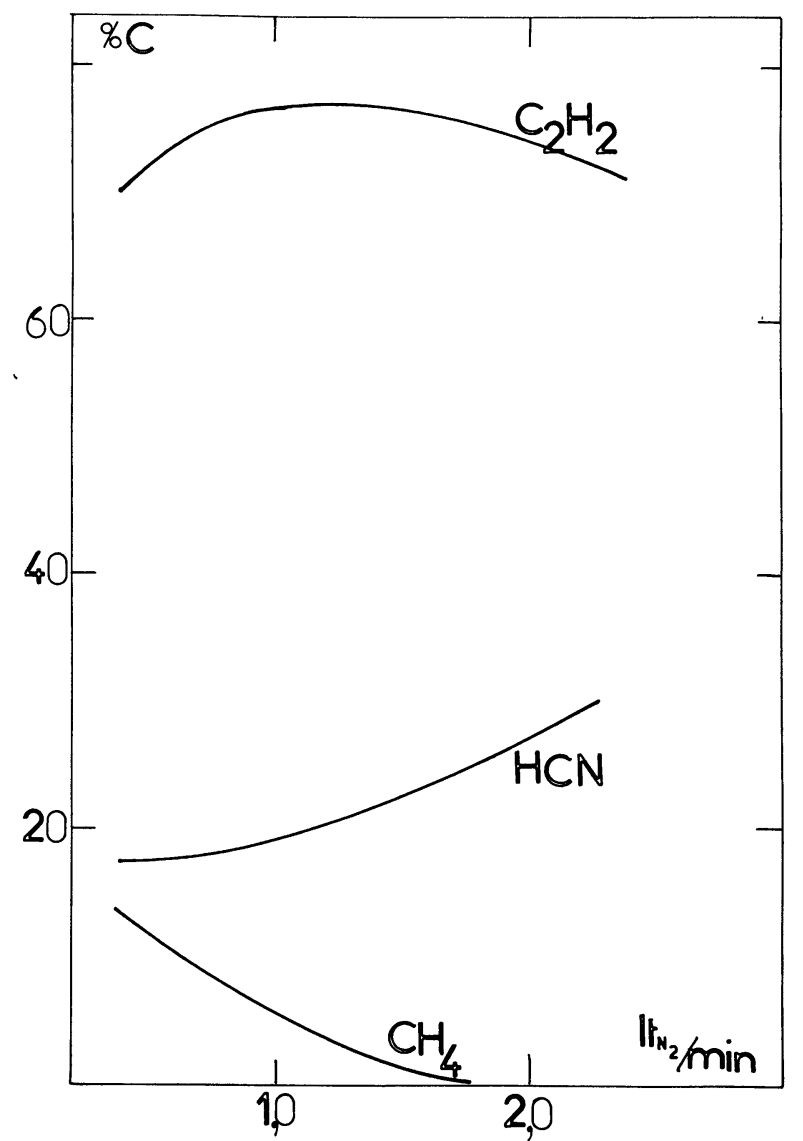

Fig. 2. - Taux de transformation du $\mathrm{CH}_{4}$ initial en $\mathrm{C}_{2} \mathrm{H}_{2}$ et $\mathrm{HCN}$ en fonction du débit de $\mathrm{N}_{2}$ exprimé $1 / \mathrm{min} . P=10-12$ torrs. Débits $\mathrm{CH}_{4}$ et énergie induite constants.

[Conversion rate of methane to $\mathrm{C}_{2} \mathrm{H}_{2}$ and $\mathrm{HCN}$ as a function of $\mathrm{N}_{2}$ flow rate.]

b) Le taux de $\mathrm{HCN}$ et de $\mathrm{C}_{2} \mathrm{H}_{2}$ reste pratiquement constant quand l'énergie par litre de $\mathrm{N}_{2}$ diminue (l'énergie totale fournie au plasma étant constante).

c) Le pourcentage de $\mathrm{N}_{2}$ fixé dỉminue rapidement mais le nombre de moles d'azote fixé par unité de temps reste constant.

Ces deux dernières constatations expérimentales montrent que le nombre de molécules $\mathrm{N}_{2}$ réactives ne varie pas notablement avec le débit de $\mathrm{N}_{2}$. Elle corroborent ainsi les conclusions de l'étude spectroscopique.

II.3 InfluenCe du DÉBIt DE $\mathrm{CH}_{4}$ (Fig. 3). - Une augmentation du débit de $\mathrm{CH}_{4}$ modifie le rendement du réacteur de trempe de la façon suivante :

a) le taux de $\mathrm{N}_{2}$ fixé sous forme de $\mathrm{HCN}$ augmente légèrement,

b) la formation de $\mathrm{HCN}$ diminue tandis que la formation de $\mathrm{C}_{2} \mathrm{H}_{2}$ augmente pour devenir majoritaire aux forts débits.

Ces résultats indiquent que la vitesse de la fixation est beaucoup plus rapide que la vitesse de transfert $\mathrm{N}_{2}^{*} \rightarrow \mathrm{CH}_{4}$. Les résultats obtenus lors de la variation de l'énergie induite vont dans le même sens. 


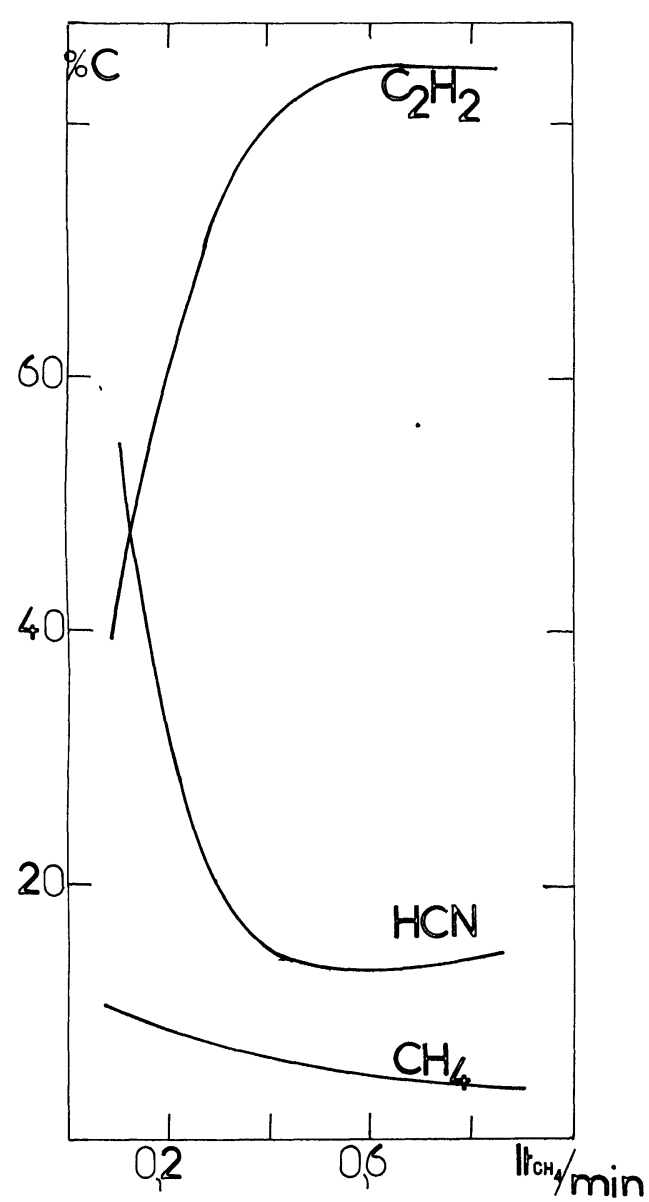

Fig. 3. - Taux de transformation du $\mathrm{CH}_{4}$ initial en $\mathrm{C}_{2} \mathrm{H}_{2}$ et $\mathrm{HCN}$ en fonction du débit de $\mathrm{CH}_{4}$. Débit $\mathrm{N}_{2}$ constant. Pression constante (11 torrs). Energie induite totale constante. Energie par litre $\mathrm{N}_{2}$ constante.

[Conversion rate of methane to $\mathrm{C}_{2} \mathrm{H}_{2}$ and $\mathrm{HCN}$ as a function of $\mathrm{CH}_{4}$ initial flow rate.]

II.4 INFLUENCE DE LA PRESSION (Fig. 4). - Les résultats montrent qu'une élévation de la pression favorise la formation de $\mathrm{HCN}$ au détriment de $\mathrm{C}_{2} \mathrm{H}_{2}$.

III. Comparaison avec l'équilibre thermodynamique. - La comparaison entre les résultats expérimentaux obtenus avec les deux types de réacteur et les équilibres calculés par la thermodynamique (Fig. 5, 6) est effectuée sur les diagrammes triangulaires portés sur les figures $7 a, b, c$. Ces diagrammes reproduisent l'évolution du taux de produits carbonés $\mathrm{C}_{2} \mathrm{H}_{2}, \mathrm{HCN}$ et $\mathrm{CH}_{4}$ (exprimés en \% du carbone total) en fonction du rapport $N / C$ et de l'énergie induite (de la température pour le calcul thermodynamique). La similitude des trois diagrammes apparaît d'une part par le domaine interdit dans la partie gauche du diagramme et d'autre part par l'évolution des courbes iso-énergie et iso-rapport analogue sur les trois diagrammes.

Bien qu'il s'agisse d'un plasma hors d'équilibre, ces conclusions ont déjà été obtenues par le système $\mathrm{CH}_{4}-\mathrm{O}_{2}$ et $\mathrm{CH}_{4}-\mathrm{Cl}_{2}[1,2]$.

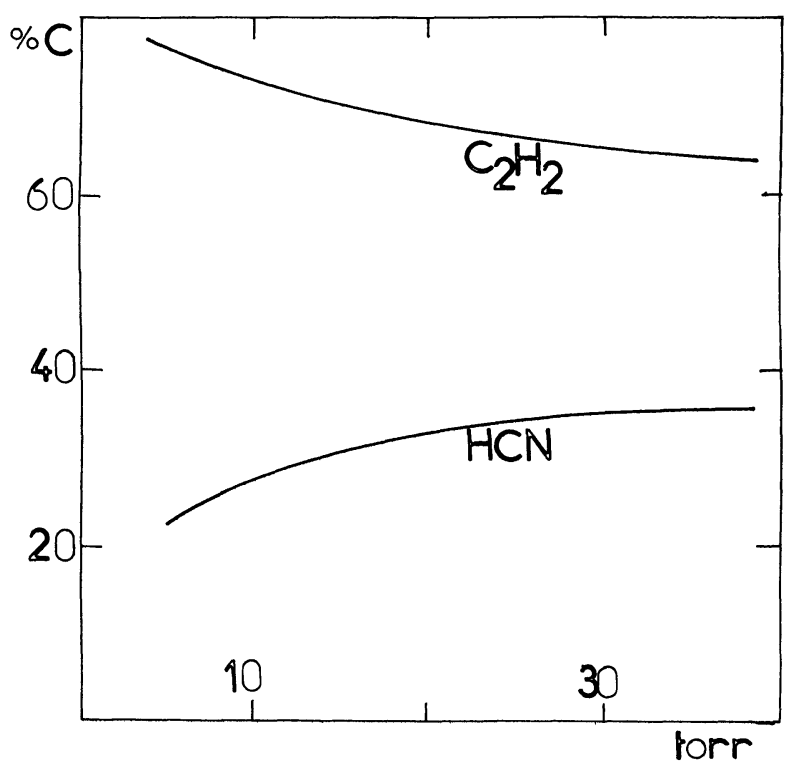

Fig. 4. - Taux de transformation du $\mathrm{CH}_{4}$ initial en $\mathrm{C}_{2} \mathrm{H}_{2}$ et $\mathrm{HCN}$ en fonction de la pression du réacteur. Débits de $\mathrm{N}_{2}$ et $\mathrm{CH}_{4}$ constants. Energie induite constante. Rapport $N / C=10$.

[Conversion rate of methane to $\mathrm{C}_{2} \mathrm{H}_{2}$ and $\mathrm{HCN}$ as a function of reactor's pressure.]

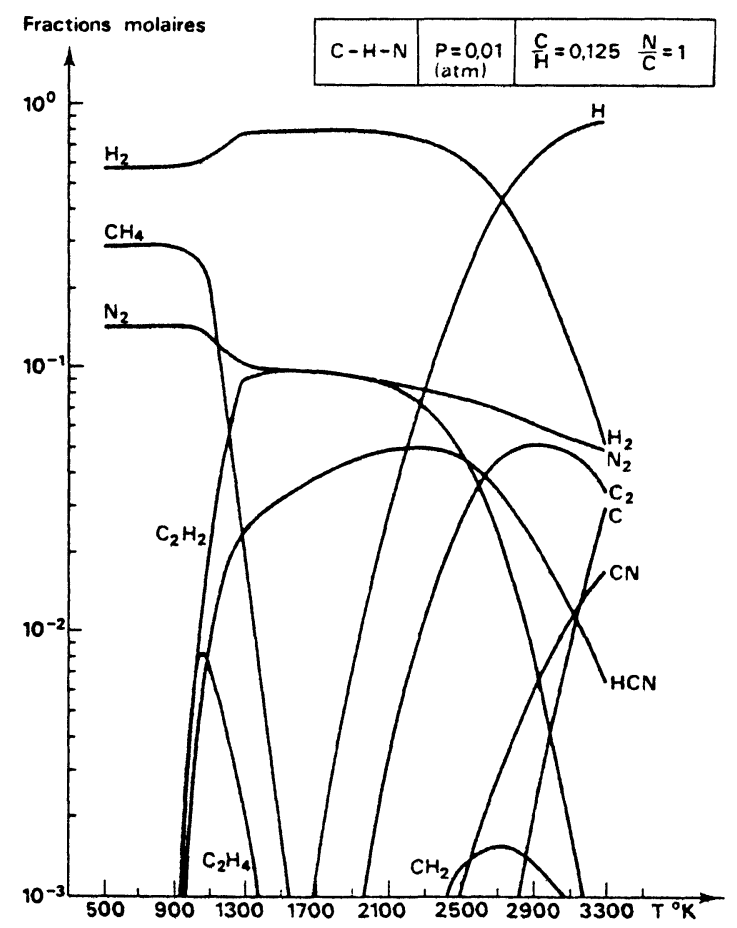

Fig. 5. - Diagramme d'équilibre thermodynamique $N / C=1,0$. [Thermodynamic equilibrium diagram $N / C=1,0$. ]

L'équilibre thermodynamique permet donc de prévoir l'évolution du système réactionnel en fonction des deux paramètres expérimentaux : composition et énergie.

Toutefois la nature hors d'équilibre du plasma écarte les résultats expérimentaux des prévisions 


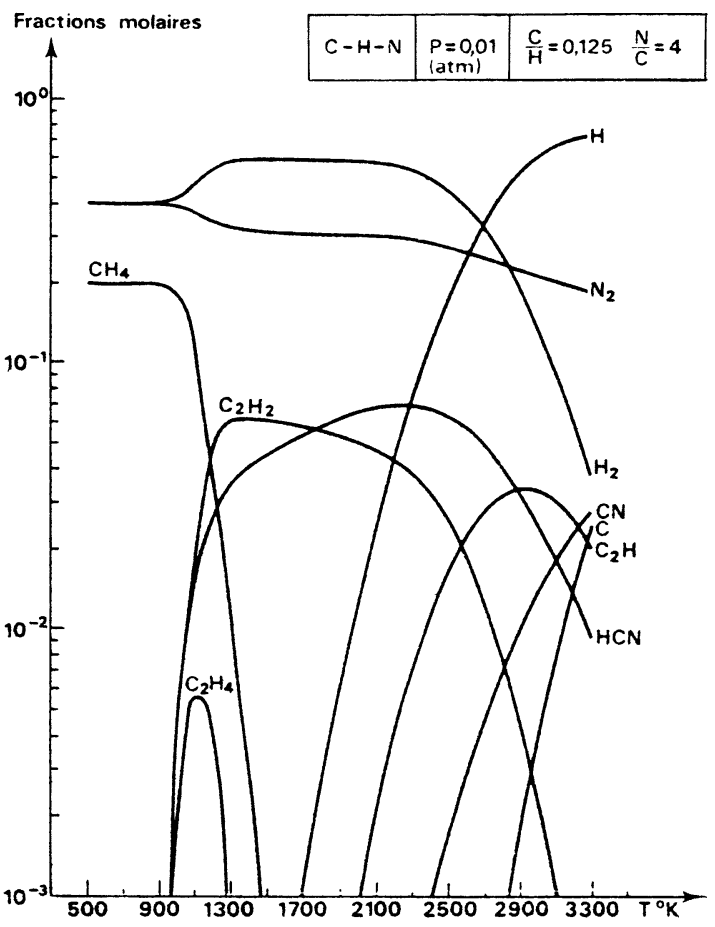

Fig. 6. - Diagramme d'équilibre thermodynamique $N / C=\Delta 0$.

[Thermodynamic equilibrium diagram $N / C=4.0$.]

thermodynamiques pour l'influence du paramètre pression. En effet, la thermodynamique prévoit une très faible influence de la pression sur la composition à l'équilibre, tandis que l'expérience le fait apparaître comme un facteur déterminant.

Ainsi nous abordons les limites de l'utilisation des calculs thermodynamiques dans la prévision de la réactivité chimique des plasmas froids. Une analyse des résultats expérimentaux par la théorie de la réactivité chimique des milieux hors d'équilibre paraît ainsi le complément nécessaire à l'étude thermodynamique et à l'étude spectroscopique.

IV. Interprétation. - Pour interpréter les mécanismes élémentaires conduisant à la fixation de l'azote et au transfert d'excitation $\mathrm{N}_{2}^{*} \rightarrow \mathrm{CH}_{4}$, nous allons examiner les états excités de $\mathrm{N}_{2}$ et de $\mathrm{CH}_{4}$, leurs processus de dissociation, ainsi que les voies possibles de transfert d'excitation.

IV.1 Excitation ET Dissociation DE $\mathrm{CH}_{4}$. Il est établi depuis plusieurs années que le spectre d'absorption de $\mathrm{CH}_{4}$ ne présente qu'une courte région continue, sans structure vibro-électronique. Le $\mathrm{CH}_{4}$ n'émet pas d'énergie lorsqu'il est soumis à l'excitation, bien que sa probabilité d'absorption est grande [6].

Dans ces conditions, il est difficile d'établir le diagramme énergétique de $\mathrm{CH}_{4}$. Ditchburn [4] suppose que tous les états électroniques excités sont dissociatifs. L'importance relative des différentes voies de dissociation a été étudiée par Polak et Slovetskii [5]
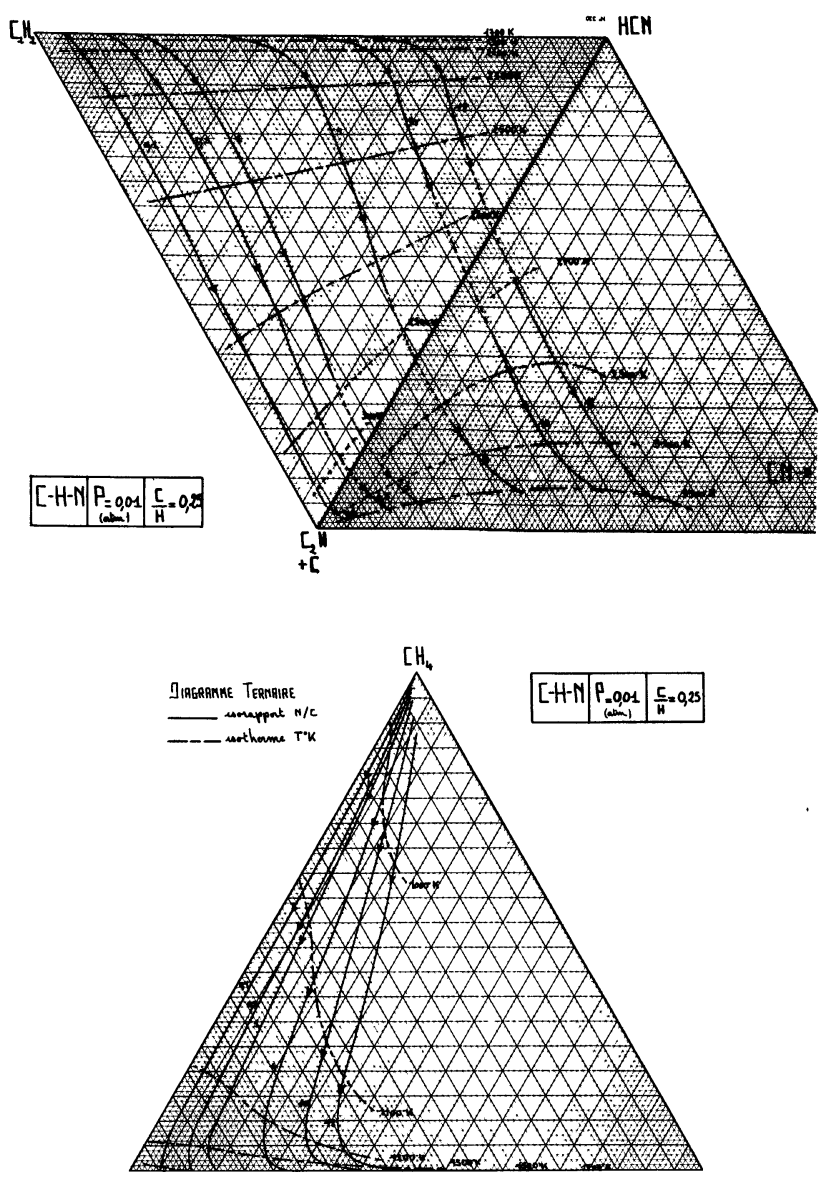

a)

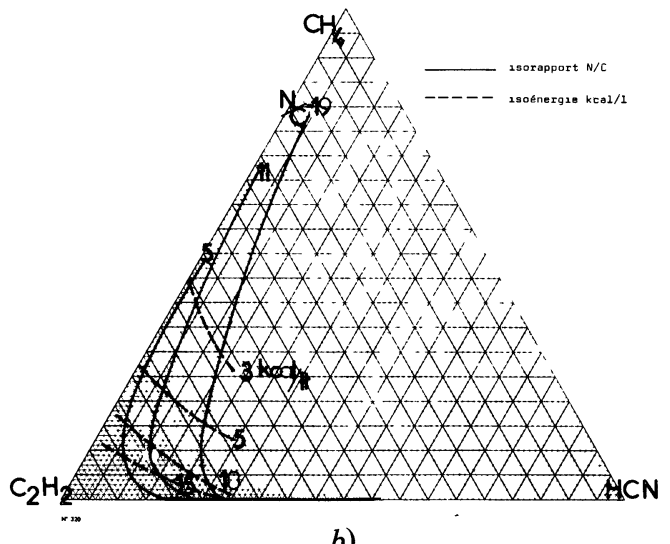

b)

Fig. 7. - Diagrammes ternaires des produits carbonés.

a) Equilibre thermodynamique.

b) Plasma $\mathrm{N}_{2}$ trempé au $\mathrm{CH}_{4}$.

[Ternary equilibrium diagram for carbon containing products.

a) Thermodynamic equilibrium.

b) Experimental.]

(Fig. 8). Winters [13] a étudié expérimentalement la dissociation de $\mathrm{CH}_{4}$ par les électrons.

Les conclusions que nous pouvons tirer de ces travaux sont les suivantes :

- Toute excitation électronique qui ne conduit pas à la dissociation, ionise la molécule $\mathrm{CH}_{4}$. 


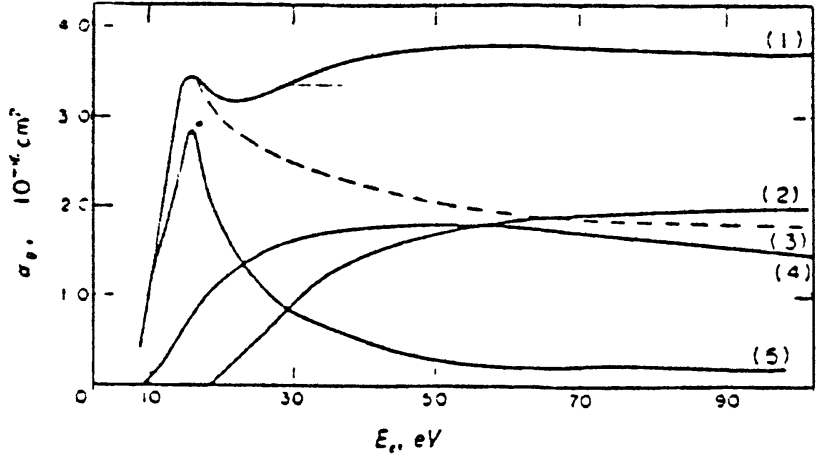

Fig. 8. - Section efficace de la dissociation de $\mathrm{CH}_{4}$ en fonction de l'énergie des électrons : 1 . - Totale, d'après les données de Manton et Tikner [14] et de Melton [15]. 2. - Ionisation dissociative. 3. - Somme de la dissociation à partir des états électroniques excités. 4. - Par excitation des transitions permises. 5. - Par excitation des transitions interdites optiquement.

[Cross section for $\mathrm{CH}_{4}$ dissociation as a function of electrons energy : 1. - Total experimental (after Manton and Tikner [14] and Melton [15]). 2. - Dissociative ionization. 3. - Sum of dissociation from excited electronic states. 4. - Dissociation by permitted transitions. 5. - Dissociation by forbitten transitions.]

- La section efficace de la dissociation est une fonction linéaire de l'énergie des électrons au voisinage du seuil énergétique d'excitation.

- Près du seuil d'excitation, l'excitation des transitions interdites est prépondérante.

- Près du seuil de dissociation, le $\mathrm{CH}_{4}$ se dissocie à des fragments neutres qui sont produits soit à l'état fondamental, soit à un niveau métastable.

IV. 2 Excitation et dissociation De $\mathrm{N}_{2}$. $-\mathrm{La}$ section efficace d'excitation de $\mathrm{N}_{2}$ par collisions électroniques est relativement grande. L'application du principe Frank-Condon montre que les niveaux vibrationnels des états électroniques excités sont peuplés directement par collisions électron-azote fondamental [3]. Au départ, ces collisions inélastiques peuplent aussi bien les états électroniques excités de même multiplicité de spin avec le fondamental, que les états triplets. Bien que les sections efficaces sont voisines, Polak [5] a montré que les transitions optiquement interdites atteignent leur maximum à des énergies 3 fois plus faibles que les transitions permises.

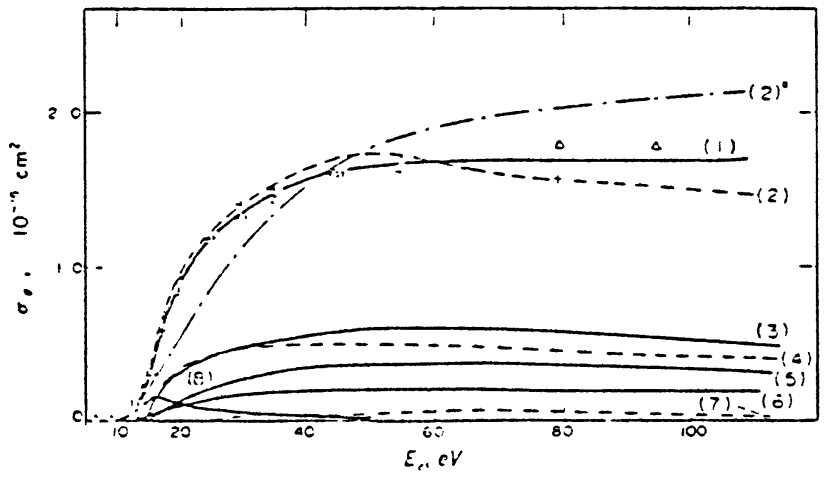

Fig. 9. - Section efficace de dissociation de la molécule $\mathrm{N}_{2}\left(\mathrm{X}^{1} \Sigma_{\mathrm{g}}\right)$, $v=0$ par collision avec des électrons. 1. - Totale, d'après les expériences de Winters $\Delta$, Niehaus + . 2. - Somme des courbes 3 à 8. 2a. - Totale, d'après les données de Brinkman et Trajmar. 3. - A travers des états de 13,6-14,4 eV. 4. - A travers des états de 12,4-13,05 eV." 5. - A travers des états de 14,41-17,7 eV. 6. - A travers des états de 13,05-13,2 eV. 7. - A travers des états $>20 \mathrm{eV}$. 8. - A travers des états de a ${ }^{1} \Pi_{\mathrm{g}}$.

[Cross section for $\mathrm{N}_{2}$ dissociation as a function of electron energy. 1. - Total experimental (Winters $\Delta$, Niehaus + ). 2. - Sum of curves 3 to 8. 2a. - Total experimental (Brinkman and Trajmar). 3. - From 13.6-14.4 eV excited states. 4. - From 12.4-13.05 eV excited states. 5. - From 14.41-17.7 eV excited states. 6. - From 13.05-13.2 eV excited states. 7. - From $>20 \mathrm{eV}$ excited states. 8. - From a ${ }^{1} \Pi_{\mathrm{g}}$ state.]

Ainsi, les états excités de $\mathrm{N}_{2}$ majoritaires dans la décharge seront les triplets $\mathrm{A}^{3} \Sigma_{\mathrm{u}}^{+}, \mathrm{B}^{3} \Pi_{\mathrm{g}}, \mathrm{C}^{3} \Pi_{\mathrm{u}}$, vibrationnellement excités.

La dissociation de $\mathrm{N}_{2}$ peut se faire soit par voie directe $\left(\mathrm{N}_{2}+\mathrm{e}^{-} \rightarrow \mathrm{N}+\mathrm{N}+\mathrm{e}\right)$. soit par étapes, à travers ces états vibro-électroniques excités, et notamment les $\mathrm{B}^{3} \Pi_{\mathrm{g}}, \mathrm{C}^{3} \Pi_{\mathrm{u}}, \mathrm{a}^{1} \Pi_{\mathrm{g}}$.

La prédissociation de ces états est facile [7]. Polak [5] a pu calculer l'importance relative des différentes voies de dissociation (Fig. 9).

En conclusion, nous pouvons admettre que dans notre réacteur la dissociation de $\mathrm{N}_{2}$ se fait par prédissociation des états vibro-électroniques $\mathrm{C}^{3} \Pi_{\mathrm{u}}$ et $\mathrm{B}^{3} \Pi_{\mathrm{g}}$, compte tenu du faible taux d'ionisation et de l'absence de raies atomiques dans le spectre d'émission.

IV.3 Transfert D'EXCitation azote-mÉTHANE. Trois réactions de transfert conduisant à la dissociation de $\mathrm{CH}_{4}$ sont possibles dans notre réacteur compte tenu de la composition du plasma d'azote :

$$
\begin{gathered}
\left.\mathrm{N}_{2}\left(\mathrm{~A}^{3} \Sigma_{\mathrm{u}}^{+}, \mathrm{v}^{\prime} \text { élevé }\right)+\mathrm{CH}_{4} \rightarrow \mathrm{N}_{2}\left(\mathrm{X}^{1} \Sigma_{\mathrm{g}}^{+}, \mathrm{v}=0\right)+\mathrm{CH}_{4}^{*} \text { (excité électroniquement }>8 \mathrm{eV}\right) \\
\left.\mathrm{N}_{2}\left(\mathrm{~A}^{3} \Sigma_{\mathrm{u}}^{+}, \mathrm{v} \text { quelconque }\right)+\mathrm{CH}_{4} \rightarrow \mathrm{N}_{2}\left(\mathrm{X}^{1} \Sigma_{\mathrm{g}}^{+}, \mathrm{v} \text { quelconque }\right)+\mathrm{CH}_{3}+\mathrm{H} \text { (état fondamental, } 4,6 \mathrm{eV}\right) \\
\left.\mathrm{N}_{2}\left(\mathrm{C}^{3} \Pi_{\mathrm{u}}, \mathrm{v}=3 \text { ou } 4\right)+\mathrm{CH}_{4} \rightarrow \mathrm{N}_{2}\left(\mathrm{~B}^{3} \Pi_{\mathrm{g}}, \mathrm{v}=0 \text { ou } 1\right)+\mathrm{CH}_{3}+\mathrm{H} \text { (états fondamentaux }\right)
\end{gathered}
$$

Pour distinguer le mécanisme le plus probable dans notre réacteur, nous allons examiner les facteurs qui déterminent l'efficacité du transfert d'excitation électronique entre molécules. a) Facteur cinétique : un grand nombre de collisions subi par le donneur d'énergie favorisera le transfert. Les réactions 1 et 2 , faisant appel à un métastab̆le, sont favorisées de ce point de vue. 
b) Facteur énergétique : une bonne efficacité du transfert nécessite la parité des transitions du donneur et du récepteur (transfert résonnant). Le recouvrement de spectre d'émission de $\mathrm{N}_{2}$ et d'absorption de $\mathrm{CH}_{4}$ est satisfaisant dans le cas de la réaction (1).

c) Facteur de structure : le transfert est plus efficace lorsque le spin total est conservé. Toutefois, lorsque la durée de vie du donneur est très longue, le transfert par une transition interdite peut être efficace.

En conclusion, nous pouvons éliminer la réaction (3) car elle est concurrencée par la prédissociation importante de $\mathrm{N}_{2}\left(\mathrm{C}^{3} \Pi_{\mathrm{u}}\right)$ et le recouvrement des spectres est mauvais. La réaction (1) est permise par le spin, mais elle a lieu à partir de niveaux vibrationnels supérieurs, peu peuplés. Elle ne participe donc que partiellement au transfert, conjointement avec la réaction (2) qui, bien qu'interdite par le spin, a lieu à partir de tous les niveaux vibrationnels de $\left(\mathrm{A}^{3} \Sigma_{\mathrm{u}}^{+}\right)$.

Ainsi, l'examen des mécanismes d'excitation, de dissociation et de transfert d'excitation nous permet d'expliquer la plus grande rapidité de la fixation de l'azote par le fait qu'elle procède à partir de niveaux vibro-électroniques très excités et très réactifs. $\mathrm{La}$ formation de $\mathrm{C}_{2} \mathrm{H}_{2}$ par transfert d'énergie est lente car elle a lieu soit pour une réaction interdite par le spin soit à partir de niveaux vibrationnels supérieurs peu peuplés.

V. Etude de la réactivité chimique du plasma hors d'équilibre. Rôle du réacteur de synthèse et du réacteur de trempe. - L'analyse spectroscopique a montré le caractère hors d'équilibre des plasmas obtenus et souligne le rôle de la pression qui permet d'accentuer ou de diminuer le caractère hors d'équilibre, selon la valeur choisie. Il est donc indispensable à partir de l'étude bibliographique et expérimentale de proposer une première approche du rôle des processus hors d'équilibre sur la réactivité chimique du mélange.

V.1 RAPPEL DE LA THÉORIE [12]. - Pour présenter un cas relativement simple nous examinerons la dynamique des réactions chimiques hors d'équilibre à l'aide de la réaction colinéaire tri-atomique.

$$
\mathrm{A}+\mathrm{BC} \rightarrow \mathrm{AB}+\mathrm{C}
$$

qui seule se prête à une représentation graphique. Le traitement de réactions comprenant plusieurs atomes est tout à fait analogue [8].

La dynamique d'une telle réaction dépend de la forme de sa surface d'énergie potentielle et plus particulièrement de la position de la barrière énergétique (qui correspond à une gorge sur cette surface $[9-10])$.

Deux cas de figures sont possibles :

1) La barrière énergétique se trouve sur la vallée d'entrée de la surface de l'énergie potentielle (Fig. 10, I). Cela signifie que l'étape la plus difficile
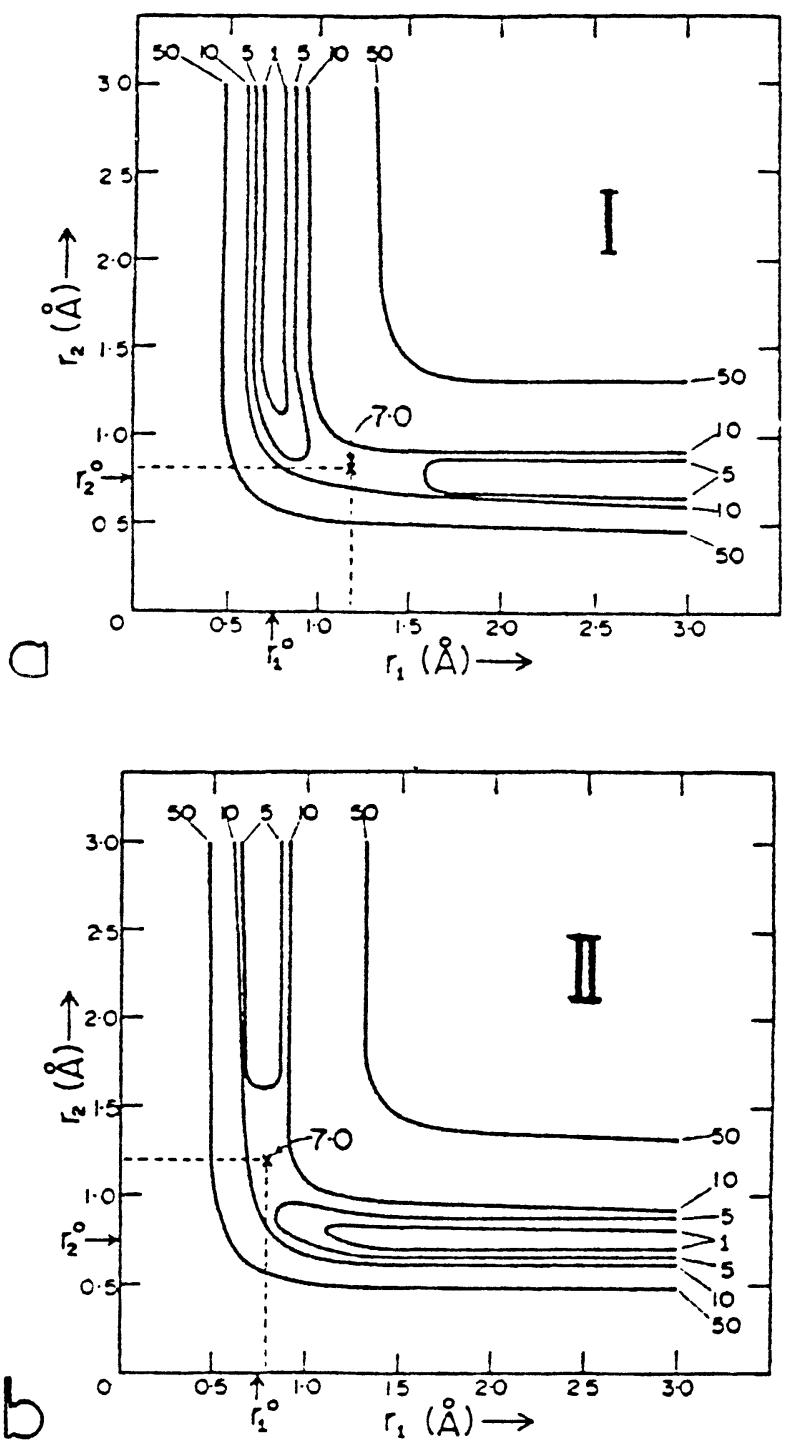

Fig. 10. - Diagramme énergétique de la réaction

$$
\mathrm{A}+\mathrm{BC} \rightarrow \mathrm{AB}+\mathrm{C} .
$$

Les courbes représentent les lignes iso-énergétiques (en $\mathrm{kcal} / \mathrm{mol}$ ) de la surface. Le sommet du chemin de moindre énergie est indiqué par un $\times$ (ici il est de $7 \mathrm{kcal} / \mathrm{mol}$ ). La distance $r_{1}$ correspond à la distance entre $\mathrm{A}$ et $\mathrm{B}$, tandis que la distance $r_{2}$ correspond à la séparation entre $B$ et $C$. La réaction se réalise par un déplacement de droite à gauche, c'est-à-dire par le rapprochement de A et B, la distance entre $B$ et $C$ ne variant pas beaucoup. Lorsque le rapprochement entre $\mathrm{A}$ et $\mathrm{B}$ commence à perturber sérieusement la liaison BC, nous approchons de la barrière énergétique. L'accomplissement de la réaction se traduit par la stabilisation de la distance A-B, tandis que la distance B-C augmente. Dans le cas (a), la barrière énergétique doit être dépassée lors du rapprochement entre A et B-C, tandis que dans le cas (b) elle doit être dépassée lors de l'éloignement entre $\mathrm{B}$ et C. $r_{1}$ et $r_{2}$ sont les distances $\mathrm{AB}$ et $B C$ sans perturbation (d'après Polanyi).

[Potential energy diagram for the reaction $\mathrm{A}+\mathrm{BC} \rightarrow \mathrm{AB}+\mathrm{C}$. Curves represents isoenergetic lines (in $\mathrm{kcal} / \mathrm{mol}$ ) of the potential energy surface. $x$ denotes the maximum of the lowest energy path (here $7 \mathrm{kcal} / \mathrm{mol}$ ). $r_{1}$ : distance between $\mathrm{A}$ and $\mathrm{B}$ atoms. $r_{2}$ : distance between $\mathrm{B}$ and $\mathrm{C}$ atoms. Reaction corresponds to a displacement from right to left. In case $(a)$ the energy barrier is located on A and B rapprochment, while in case $(b)$ it is located on $B$ and $C$ separation.] 
(étape-clé) est le rapprochement de A et de BC, c'est-à-dire l'amorçage de la nouvelle liaison à créer.

2) La barrière énergétique se situe sur la vallée de sortie de la surface d'énergie potentielle (Fig. 10, II). Dans ce cas, l'étape difficile est la séparation de B et de C, c'est-à-dire la rupture de la molécule de départ.

La sélectivité du mode d'excitation de la molécule des réactifs $\mathrm{BC}$ est différente dans les deux cas :

L'excitation vibrationnelle favorise la réaction dans le cas de la surface de type II, tandis qu'elle inhibe la réaction dans le cas de la surface de type I. La situation inverse se produit lorsque l'énergie est canalisée sur la rotation-translation des réactifs.

Cette analyse de la dynamique des réactions hors d'équilibre serait de peu d'intérêt pratique si, pour chaque réaction, nous devions chercher le type de surface énergétique approprié. Heureusement tel n'est pas le cas, car les réactions exothermiques correspondent à des surfaces d'énergies potentielles de type $\mathrm{I}$, tandis que les réactions endothermiques à des surfaces de type II. Nous sommes ainsi en mesure de prévoir le mode d'excitation sélectif le plus efficace dans le cas des réactions chimiques ayant une enthalpie de réaction significative.

V.2 DynamiQue de la RÉACTION CHIMIQue DANS UN PLASMA. - Dans le cas où la réaction

$$
\mathrm{A}+\mathrm{BC}^{*} \rightarrow \mathrm{AB}+\mathrm{C}
$$

a lieu dans un plasma basse pression, nous pouvons distinguer trois étapes (Fig. 11).

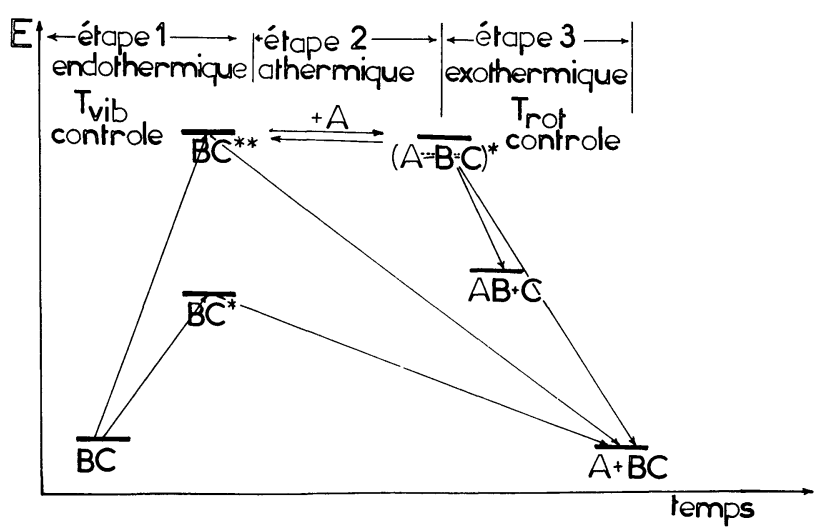

Fig. 11. - Diagramme d'évolution d'une réaction en phase plasma. [Energy diagram of the plasma phase $\mathrm{BC}^{*}+\mathrm{A} \rightarrow \mathrm{AB}+\mathrm{C}$ reaction.]

- Première étape : excitation des réactifs par la décharge.

La molécule initiale $\mathrm{BC}$ est excitée soit à l'état $\mathrm{BC} * *$ contenant suffisamment d'énergie pour rompre la liaison $\mathrm{B}-\mathrm{C}$, soit à l'état $\mathrm{BC}^{*}$ moins énergétique qui ne contient pas assez d'énergie pour se dissocier.
Il s'agit d'une étape endothermique favorisée par une température de vibration élevée.

- Deuxième étape : recombinaison entre les atomes du système réactionnel (réacteur de synthèse).

Aucun apport ou dépense d'énergie n'est nécessaire, et de ce point de vue la réaction est réversible.

- Troisième étape : stabilisation (réacteur de trempe).

Elle contient un excès d'énergie (énergie de la liaison formée) qui doit être évacuée rapidement (trempe) pour éviter sa décomposition. Il s'agit donc d'un processus exothermique, favorisé par une température de translation-rotation élevée.

Ainsi la vitesse de la réaction globale dépend des vitesses $\left(v_{1}, v_{2}\right.$ et $\left.v_{3}\right)$ de chacune des trois étapes :

V.3 APPLICATION AUX SYSTÈMES EXPÉRIMENTAUX $\mathrm{CH}_{4}-\mathrm{N}_{2}$ ET $\mathrm{O}_{2}-\mathrm{N}_{2}$. - a) Cas du système $\mathrm{N}_{2}+\mathrm{CH}_{4}$ : La réaction de la fixation de l'azote

$$
\mathrm{N}_{2}^{* *}+\mathrm{CH}_{4} \rightarrow\left(\mathrm{N}_{\ldots} \mathrm{C}_{\ldots} \mathrm{H}\right)_{X}^{*} \rightarrow \mathrm{HCN}+(X-1) \mathrm{H}
$$

aura lieu selon le diagramme suivant (Fig. 12).

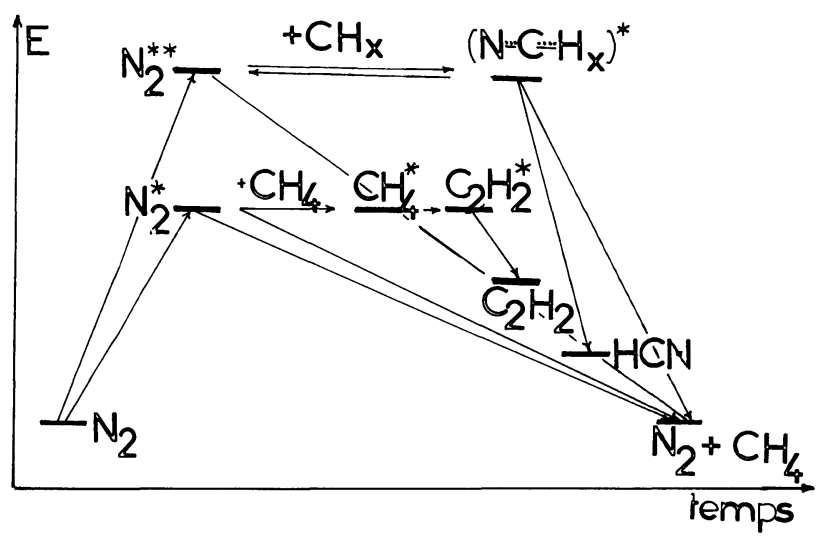

Fig. 12. - Diagramme énergétique de la réaction $\mathrm{N}_{2}+\mathrm{CH}_{4}$

[Energy diagram for $\mathrm{N}_{2}+\mathrm{CH}_{4}$ reaction.]

La nouvelle molécule $\left(\mathrm{N}_{\ldots}-\mathrm{C}_{\ldots}-\mathrm{H}_{X}\right)^{*}$ contient un important excès d'énergie qui varie de 37 à $145 \mathrm{kcal} / \mathrm{mole}$ (selon la valeur de $x$ ) ou même plus si $\mathrm{N}_{2}^{* *}$ est excité au-delà du seuil de dissociation de $225 \mathrm{kcal} / \mathrm{mole}$ (c'est le cas de l'état $\mathrm{C}^{3} \Pi_{\mathrm{u}}$ ).

L'évacuation de cet excès d'énergie est l'étape difficile, puisque c'est l'acheminement de l'énergie induite vers la rotation-translation qui favorise la fixation expérimentale de $\mathrm{N}_{2}$. Il apparaît ainsi que la trempe par collision avec la paroi ou avec des molécules froides joue un rôle déterminant.

Ainsi la réaction exothermique de désexcitation de l'espèce $(\mathrm{HCN}) *$ contrôle le taux de formation de la molécule recherchée $\mathrm{HCN}$. L'augmentation de la pression du réacteur à énergie induite constante, favorise la fixation de $\mathrm{N}_{2}$, car elle augmente la température de rotation-translation des gaz. 
b) Cas du système $\mathrm{N}_{2}-\mathrm{O}_{2}$. - La fixation de l'azote se fait par deux processus différents :

- réaction de l'azote dans un état pré-dissociatif et l'oxygène froid

$$
\mathrm{N}_{2}^{* *}+\mathrm{O}_{2} \rightarrow\left(\mathrm{N}_{\ldots} \mathrm{N} \ldots \mathrm{O} \ldots \mathrm{O}\right)^{*} \rightarrow \mathrm{NO},
$$

- réaction de l'azote moins excité et d'oxygène lui aussi excité par la décharge

$$
\mathrm{N}_{2}^{*}+\mathrm{O}_{2}^{*} \rightarrow(\mathrm{N} \ldots \mathrm{N} \ldots \mathrm{O} \ldots \mathrm{O})^{*} \rightarrow \mathrm{NO}
$$

ou

$$
\mathrm{N}_{2}^{*}+\mathrm{O} \rightarrow\left(\mathrm{N}_{2} \mathrm{O}\right)^{*} \rightarrow \mathrm{NO}+\mathrm{N} .
$$

Le diagramme correspondant est illustré sur la figure 13.

Nous avons constaté qu'une diminution de la $T_{\mathrm{v}}$ produit une baisse de la teneur en azote fixe. Ainsi l'étape-clé est l'étape endothermique contrôlée par la $T_{\mathrm{V}}$ c'est-à-dire celle qui forme l'espèce de type $\mathrm{N}_{2} \mathrm{O}^{*}$ ou $\left(\mathrm{N}_{-} \mathrm{N}_{-} \mathrm{O} . . \mathrm{O}\right)$ selon des mécanismes pro-

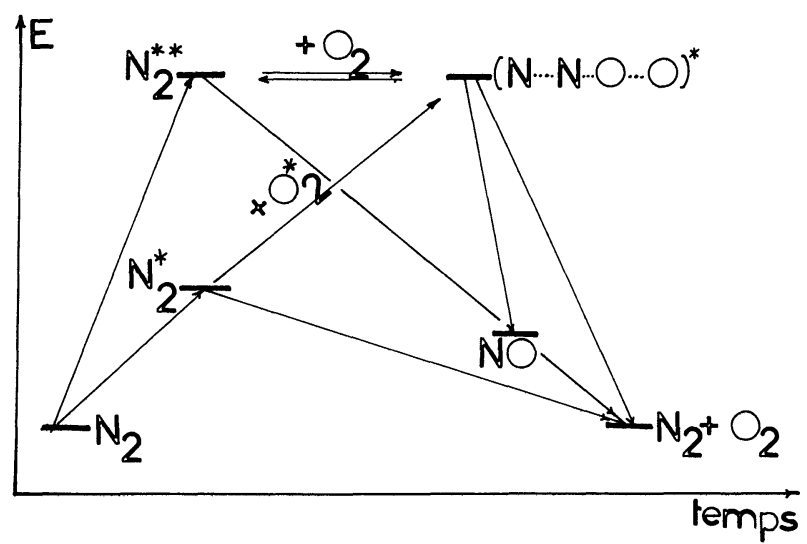

Fig. 13. - Diagramme énergétique de la réaction $\mathrm{N}_{2}+\mathrm{O}_{2}$.

[Energy diagram for $\mathrm{N}_{2}+\mathrm{O}_{2}$ reaction.] posés par Russanov [11]. Enfin l'étape ultime de désexcitation est plutôt une étape de paroi (réacteur de trempe) dont la vitesse reste toujours grande par rapport à la formation de l'espèce complexe.

La diminution de la pression augmente la $T_{\text {vib }}$. Elle favorisera l'étape endothermique, donc la fixation de l'azote sous forme de NO.

Conclusion. - La théorie des réactions chimiques dans les milieux hors d'équilibre appliquée dans le cas d'un plasma d'azote nous permet de distinguer deux étapes clés pour la transformation chimique globale. Une étape endothermique correspondant à la décomposition des réactifs et favorisée par la canalisation de l'énergie induite vers la vibration des réactifs, et une étape exothermique correspondant à la stabilisation par trempe des produits de la réaction favorisée cette fois-ci par l'élévation de la température de rotation-translation du milieu réactionnel. L'augmentation de la pression du réacteur augmente la température de rotation-translation, au détriment de la température de vibration. Une pression élevée favorisera ainsi des réactions contrôlées par une étape exothermique et inversement.

La fixation de l'azote dans le système $\mathrm{N}_{2}-\mathrm{O}_{2}$ est contrôlée par la température de vibration du réacteur de synthèse, ce qui s'explique par la participation à la formation de $\mathrm{NO}$ des états peu excités de $\mathrm{N}_{2}$ et dont la dissociation est endothermique.

Par contre dans le cas du système $\mathrm{N}_{2}-\mathrm{CH}_{4}$, la fixation de l'azote a lieu uniquement à partir d'états vibro-électroniques contenant l'énergie nécessaire à la dissociation de $\mathrm{N}_{2}$ (les états excités de $\mathrm{N}_{2}$ de faible énergie servent à dissocier le $\mathrm{CH}_{4}$ par transfert d'énergie). Ainsi le réacteur de trempe, c'est-à-dire l'étape d'évacuation de l'excès d'énergie ainsi accumulé sur les produits de la réaction, devient l'étape déterminante. Elle est contrôlée par la température de rotation-translation du système chimique.

\section{Bibliographie}

[1] Amouroux, J., Foll, J.-P., Rapakoulias, D., Ann. Chim. 9 (1974) 181.

[2] Rapakoulias, D., Amouroux, J., Ann. Chim. 8 (1973) 193.

[3] Borst, W. L., Chang, S. L., J. Chem. Phys. 59 (1973) 5830.

[4] Ditchburn, A., Proc. R. Soc. 229A (1955) 44.

[5] Polak, L. S., SlovetskiI, D. I., Int. J. Radiat. Phys. Chem. 8 (1976) 257.

[6] Hertzberg, G., Electronic Spectra of Polyatomic Molecules (Van Nostrand) 1966.

[7] Graydon, A. G., Dissociation Energies and spectra of diatomic molecules (Chapmann \& Hall) 1968.

[8] Ashmore, P. G., ed. Gas kinetics and energy transfer, The Chemical Society, 1977.
[9] Polanyi, J. C., Wong, W. H., J. Chem. Phys. 51 (1969) 1439.

[10] Polanyi, J. C., Acc. Chem. Res. 5 (1972) 161.

[11] Russanov, V. D., Fridmann, A. A., Dokl. Akad Nauk SSSR. 231 (1976) 1109.

[12] Rapakoulias, D., Thèse, Université Pierre-et-Marie-Curie (Paris VI), 1979.

[13] Winthers, H. F., J. Chem. Phys. 63 (1976) 3462.

[14] Manton, J. E., Tikner, A. W., Can. J. Chem. 38 (1960) 858.

[15] Melton, C., Rudolph, P. S., J. Chem. Phys. 47 (1967) 1771

[16] Niehaus, A., Z. Naturforsch. 22A (1967) 690.

[17] Brinkmann, R. T., Trajmar, V. L., Ann. Geophys. 28 (1970) 201. 